\title{
Belphégor
}

\section{Incidence des supports dans les mutations des imaginaires sériels}

Le cas d'Eichler et des dime novels européens

\section{Matthieu Letourneux}

\section{(Q) OpenEdition}

\section{Journals}

Édition électronique

URL : https://journals.openedition.org/belphegor/2629

DOI : $10.4000 /$ belphegor.2629

ISSN : 1499-7185

Éditeur

LPCM

Référence électronique

Matthieu Letourneux, « Incidence des supports dans les mutations des imaginaires sériels », Belphégor [En ligne], 18-1 | 2020, mis en ligne le 17 février 2020, consulté le 30 juin 2021. URL : http:// journals.openedition.org/belphegor/2629; DOI : https://doi.org/10.4000/belphegor.2629

Ce document a été généré automatiquement le 30 juin 2021.

\section{$\Theta \Theta \Theta \Theta$}

Belphégor est mis à disposition selon les termes de la Licence Creative Commons Attribution - Pas d'Utilisation Commerciale - Pas de Modification 4.0 International. 


\title{
Incidence des supports dans les mutations des imaginaires sériels
}

Le cas d'Eichler et des dime novels européens

\author{
Matthieu Letourneux
}

1 Lorsqu'il s'agit d'étudier la mondialisation des imaginaires, les études littéraires mettent souvent l'accent sur la circulation des œuvres, des auteurs et des personnages, bref, elles en restent à une étude des textes, indépendamment de leurs conditions concrètes de production, de circulation et de réception. Or, face aux objets de la culture de masse, une telle perspective n'est guère satisfaisante. En effet, dans la mesure où de telles productions s'inscrivent dans des logiques sérielles qui les portent et qu'elles convoquent, il convient moins de s'interroger sur la circulation des textes et leur négociation avec ceux produits par la culture qui les importe, que sur celle des séries elles-mêmes. On gagne alors à prendre du champ par rapport aux œuvres et à ressaisir les échanges non seulement au niveau du mode d'expression concerné (pour nous, la littérature) mais aussi des médias dans leur matérialité et leurs usages et, par-delà, de l'ensemble du champ médiatique (au XIXe siècle, le théâtre, les spectacles, l'édition et la presse ; au XXe siècle, le cinéma, les nouveaux modes d'expression de l'imprimé, plus tard la télévision, etc.). Pour le dire plus simplement, si, dans les imaginaires de la culture populaire, ce qui importe, ce sont les dynamiques architextuelles et leur formulation dans les séries culturelles et médiatiques, alors la question de la circulation internationale des imaginaires et de leur mondialisation doit se penser à un niveau plus global, en jouant en permanence entre le texte et son contexte matériel, parce que c'est ce contexte, autant que le texte, qui produit de la signification.

2 C'est ce que montre l'exemple de l'arrivée des dime novels en Europe au début du XXe siècle, qui ont imposé partout en quelques années des genres de récits et des formats spécifiques, et ont suscité le même type de réaction dans la plupart des pays. Un tel phénomène ne peut se comprendre si on ne l'interprète pas globalement, au niveau diégétique, narratif et médiatique, et si on ne le saisit pas dans le contexte plus large des transformations des imaginaires culturels. 


\section{La vague des dime novels européens, premier exemple d'un imaginaire industriel mondialisé}

3 On peut rapidement rappeler les circonstances de ce premier processus d'importation des imaginaires américains en Europe ${ }^{1}$. En 1904, Alwin Eichler entre en relation avec le New-Yorkais Street and Smith, l'un des plus puissants éditeurs de dime novels avec Beadle and Adams et Frank Tousey. Il lui achète les droits exclusifs de deux séries à succès décrivant les exploits de personnages récurrents, Buffalo Bill et Nick Carter, qui paraissent de manière hebdomadaire. Toutes deux ont épousé les transformations de l'édition populaire américaine. Le personnage de Nick Carter apparaît d'abord en 1886 dans le New York Weekly, un des nombreux «story papers" (équivalents de nos journaux-romans), dans un feuilleton intitulé The Old Detective's Pupil ; or, The Mysterious Crime of Madison Square. Il n'est alors que le faire-valoir d'un des héros privilégiés par ce type de supports à l'époque, le vieux détective griméz. Mais son succès conduit très vite son éditeur à lui associer un périodique qui porte son nom, le Nick Carter Detective Library (1891), puis le New Nick Carter Library et le New Nick Carter Weekly (1903). C'est ce dernier périodique, forme tardive et grand format du dime novel, qu'Eichler importe en Europe. Quant à Buffalo Bill, sa carrière débute plus tôt encore, puisque le premier récit à lui être consacré, Buffalo Bill, The King of the Border Men, écrit par le prolifique Ned Buntline, est publié dans le New York Weekly en 1869, vingt ans avant The Old Detective's Pupil. Le personnage de Buffalo Bill paraît alors tour à tour chez Street and Smith et chez Beadle and Adams, avant de se fixer chez le premier pour un nombre considérable d'aventures. On trouve des collections capitalisant sur le personnage dès les années 1880, avec par exemple les Buffalo Bill Wild West Series (1887). La collection qu'a rachetée Eichler semble être les Buffalo Bill Stories, lancées en $1901^{3}$. Autrement dit, dans les deux cas, ce que reprend Eichler, ce sont des séries aux personnages déjà bien implantés dans la culture populaire américaine, puisqu'ils ont tous deux déjà vécu une centaine d'aventures, et dont le succès s'est construit, progressivement, sur un quart de siècle. En revanche, les collections dont Eichler rachète les droits sont, elles, récentes, puisqu'elles ont respectivement un et quatre ans quand l'éditeur de Dresde lance les séries en Allemagne, en 1905. Du même coup, il existe un écart sensible entre l'effet de synchronicité produit par l'arrivée de collections normées en Allemagne puis en France et partout en Europe, et la situation d'imaginaires qui se construisent sur le long cours aux États-Unis. Certains pays d'Europe, comme l'Angleterre (de façon massive), la France, l'Allemagne ou la Suède, ont d'ailleurs déjà connu une diffusion de dime novels, et l'imaginaire de l'Ouest leur est familier. Cette tension entre nouveauté et familiarité est l'un des traits définitoires de l'arrivée de ces productions en Europe, on le verra, et contribue largement à la rapidité de leur pénétration.

4 Deux mots à présent d'Eichler. Alwin Eichler aurait d'abord travaillé chez l'éditeur allemand Munchmeyer, qui possédait des bureaux à New York, ce qui explique sans doute sa connaissance des productions américaines. Il fonde ensuite une maison d'édition de partitions de musique ${ }^{4}$. Un an après avoir racheté les droits des deux séries, il lance en Allemagne la collection des Buffalo Bill en 1905, et l'année suivante, celle des Nick Carter. La réussite est immédiate, avec des tirages hebdomadaires de 80000 exemplaires $^{5}$. En 1907, les deux séries sont lancées par Eichler en France avec le succès que l'on connaît, puis dans différents pays d'Europe, comme les Pays Bas (1907), l'Italie, le Danemark, la Pologne, la Hongrie et la Bohème (1908) pour Buffalo Bill, à 
peine moins pour Nick Carter ${ }^{6}$. La rapidité de la diffusion de l'œuvre surprend jusqu'aux Américains eux-mêmes, qui consacrent au phénomène plusieurs articles oscillant entre fierté et ironie ${ }^{7}$. Mais le succès des deux séries ne s'arrête pas aux productions d'Eichler. Elles sont en effet rapidement diffusées dans la plupart des pays d'Europe, parfois dans des versions qui n'ont plus grand-chose à voir avec les textes américains ${ }^{8}$. C'est un véritable raz-de-marée qui traverse toute l'Europe, n'épargnant quasiment aucun pays, central ou périphérique ${ }^{9}$. Et c'est sans doute le premier exemple d'une circulation synchronique, dans presque toute l'Europe, d'une série romanesque et d'un type de support.

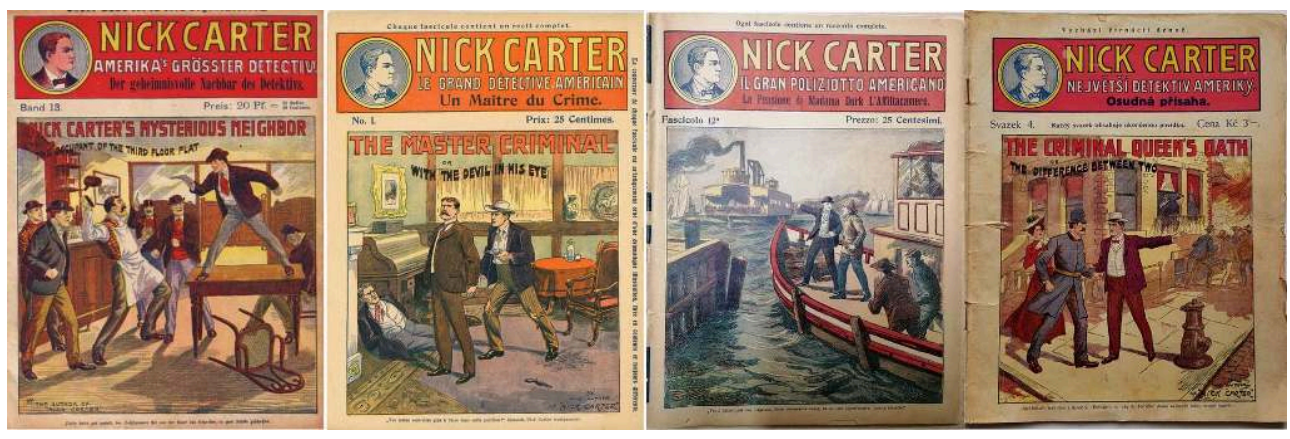

Images 1 à 4 . Légendes : Éditions allemande, française, italienne et tchèque de Nick Carter. Les effets de standardisation d'un pays à l'autre sont sensibles dès la couverture.

Cette circulation exceptionnelle ne peut se comprendre que si l'on tient compte de la spécificité du support. Extrêmement séduisant par son américanité et sa modernité ostentatoire, le dime novel retrouve en réalité des formats déjà bien implantés dans la plupart des pays européens : livraisons françaises, allemandes ou italiennes ${ }^{10}$, penny dreadfuls britanniques, fascicules et livrets vendus en colportage un peu partout. Dès lors, ces périodiques à la fois modernes et archaïques peuvent facilement occuper les réseaux traditionnels de l'édition populaire: kiosques à journaux, merceries ${ }^{11}$, marchands de tabac ${ }^{12}$, marchés, colportage ${ }^{13}$, etc. C'est sans doute parce que, tout en apparaissant résolument moderne, le dime novel s'est intégré à des pratiques autochtones bien implantées, qu'il a pu si bien circuler en Europe, y compris dans des pays culturellement périphériques ${ }^{14}$. C'est ce que montre par exemple le cas de la Bohème, future Tchécoslovaquie, dont les éditeurs populaires se sont rapidement appropriés le support bon marché du dime novel (dans un format réduit plus économique encore), pour distribuer les aventures d'épigones qu'on appellera les « cliftonky $»^{15}$. Dans tous les cas cependant, l'arrivée des fascicules est vécue comme un véritable choc par rapport aux pratiques du colportage et de l'édition populaire, tant la force de frappe industrielle modifie en profondeur la relation à ces publications. En rationalisant les modes de production et de distribution des imprimés populaires, et en leur donnant une ampleur internationale, Eichler a puissamment contribué à faire basculer dans de nombreux pays l'édition populaire dans une nouvelle dimension économique et culturelle ${ }^{16}$. 




Image 5 : Un kiosque à journaux, 1910. En haut à gauche, on peut identifier les publications Eichler.

Certes, le succès foudroyant d'Eichler a sans doute tenu en partie de la cavalerie, puisque l'éditeur se suicide en 1912, ne pouvant payer ses créanciers. Mais il n'empêche que sa réussite est aussi industrielle : c'est celle d'une des premières grandes maisons d'édition conçue comme une multinationale. Qu'Eichler ait cherché à tirer parti de cette licence européenne pour offrir rapidement un même produit dans plusieurs pays d'Europe témoigne de ce qu'il avait compris que la logique de la culture de masse est aussi une logique d'échelle. Suivant une dynamique de standardisation du produit qui anticipe sur le fordisme industriel, Eichler achète non seulement des textes, mais aussi un format, en prenant soin d'acquérir en même temps les illustrations de couverture, reproduites à l'identique dans tous les pays, et une forme éditoriale, l'hebdomadaire romanesque de récits complets à personnage récurrent. En Allemagne, il a recours à un réseau extrêmement structuré. Si l'on en croit Georg Jäger (op. cit.), dans le cas de l'Allemagne et de l'Autriche, le nombre de libraires distribuant ces fascicules s'élevait à 8000 , quant aux colporteurs, ils auraient été 30000 , voire $50000^{17}$. Mais la dynamique industrielle affecte également la politique éditoriale, qui repose sur un principe de sérialisation jouant à tous les niveaux. Non seulement l'éditeur publie les deux séries dans plusieurs pays, mais il va rapidement les décliner dans des productions similaires. En France par exemple, Buffalo Bill donne les séries Texas Jack ou Sitting Bull ${ }^{18}$; et Nick Carter est décliné en Ethel King ("le Nick Carter féminin ») et Nat Pinkerton (tous écrits par des auteurs allemands). L'éditeur explore aussi des principes de complémentarité, lançant des séries présentant des bandits d'honneur (Claude Duval) ou des aventures pirates (Stoerte-Becker). La plupart du temps, Eichler rachète des séries allemandes qu'il diffuse dans les autres pays. Mais la multinationale fonctionne de façon globale: l'éditeur distribue également des productions venues d'Angleterre, comme les aventures de Claude Duval et de Dick Turpin (dans la version de Charlton Lea), ou importées d'Italie, comme celle du Vengeur (sans doute Sangue Siciliano de Guido Bassi). 
C'est là l'étonnante modernité d'Eichler que de concevoir une véritable industrie européenne, dans laquelle l'éditeur ne publie quasiment aucun texte original, mais se contente de traduire, d'un pays à l'autre, la matière qu'il rachète à d'autres. Les contemporains ont été sensibles au caractère mondialisé de ces périodiques qui a contribué à nourrir leurs inquiétudes. De fait, c'est aussi l'apparition d'une véritable industrie culturelle multinationale - pire, allemande ! - qui effraie. C'est en tout cas ce que semblent montrer dans la presse les articles évoquant les réactions négatives, d'un pays à l'autre, au développement de ces publications ${ }^{19}$.

\section{Américanité et modernité}

7 Reste à comprendre le choix qui est fait des aventures de Buffalo Bill et de Nick Carter. Avant d'aller acheter ces séries aux États-Unis, Eichler s'intéressait déjà à la figure de Buffalo Bill, au point de demander en 1900-1901 à Karl May, le fameux auteur de romans de l'Ouest, de lui écrire un récit consacré au personnage. Mais malgré ses promesses, Karl May n'en fera rien ${ }^{20}$. Autrement dit, en rachetant des collections de dime novels aux États-Unis, Eichler ne vise pas à importer un nouveau genre de récits. Au contraire, il tente de capitaliser sur le succès d'un genre allemand, celui du roman de l'Ouest, dont Karl May figure alors comme l'incontestable référence, et Möllhausen et Gerstäcker, les précurseurs. En décidant de traduire des romans américains, Eichler répond ainsi au succès de Karl May. Mécontent de ses relations avec le romancier allemand, il oppose même peut-être à ce voyageur de pacotille qu'est Karl May son double authentique (mais tout aussi féru d'autopromotion) qu'est le colonel Cody. Plus probablement, il cherche à nourrir, à bon marché, les appétits des lecteurs allemands pour ce type de récits.

8 Le choix du personnage de Buffalo Bill plutôt que d'un autre héros de frontier stories n'est cependant pas anodin. Dix ans plus tôt, en 1890, le Colonel Cody a fait sensation en Allemagne à la suite d'une tournée européenne du Wild West Show durant laquelle il a également séduit les Anglais, les Français et les Italiens ${ }^{21}$. Il est revenu en Allemagne, en France et dans toute l'Europe lors de sa tournée de 1903-1906, qu'il a présentée comme une tournée d'adieux. Autrement dit, la série des Buffalo Bill est importée au moment même où les Européens le (re)découvrent «en chair et en os ", ce qui produit un fort effet de réel. C'est donc pour cette même raison de synchronie qu'on peut comprendre le succès rencontré tout de suite par les fascicules de Buffalo Bill. Publier Buffalo Bill, c'est importer l'Amérique réelle.

9 Mais ce qui a ébloui les Européens à travers le Wild West Show de Buffalo Bill c'est également l'efficacité de la machine médiatique, avec ses trains décorés, ses nombreux navires affrétés, ses affiches placardées partout sur les murs de la ville, ses parades démesurées, et sa façon d'orchestrer l'exceptionnel avec des méthodes dignes de Barnum $^{22}$. Buffalo Bill importe des méthodes promotionnelles inédites en Europe. Ainsi mêle-t-il les deux motifs associés traditionnellement à l'Amérique, celui d'une terre sauvage à coloniser (l'Amérique de la frontier et du Wild West Show) et celui d'une nation moderne, technique et industrielle, qu'incarnent ses méthodes promotionnelles et sa machinerie spectaculaire. Buffalo Bill lui-même conçoit ses spectacles comme de véritables démonstrations de puissance ; il s'est constitué consciemment en personnage de fiction, usant des techniques de communication les plus modernes, comme la publicité et le cinématographe ${ }^{23}$. Autrement dit, le monde de Buffalo Bill est moderne. 
10 La situation de Nick Carter peut être rapprochée de celle de Buffalo Bill. Il est importé par Eichler comme un épigone d'un autre personnage à succès, Sherlock Holmes. Mais comme pour Buffalo Bill, Eichler exploite à travers lui des traits associés à l'imaginaire américain. De fait, comme l'a montré Dominique Kalifa ${ }^{24}$, le détective privé américain est également une figure familière depuis au moins une décennie, à travers les activités de l'agence Pinkerton, fréquemment relayées dans la presse. En outre, cette célébrité est souvent associée à un imaginaire du battage publicitaire - c'est ce qui apparaît en 1906, dans Le Voleur d'enfants de Louis Forest, qui montre un détective américain, William Trisson, plus soucieux d'autopromotion que d'enquête rigoureuse, et ce sera encore le cas dans Fantômas quand on évoquera en 1911 le détective Tom Bob, venu des États-Unis faire sa promotion ${ }^{25}$.

11 En exploitant la vogue du détective et celle du Wild West Show, Eichler capitalise sur un goût pour les imaginaires américains qui dépasse largement le cas des deux séries importées. C'est ce qui explique que ces dernières aient pu susciter un nombre incalculable d'épigones dans tous les pays d'Europe. Cow boys et détectives américains de pacotille se multiplient partout, au point qu'il serait fastidieux de les énumérer ${ }^{26}$. Mais quelques exemples de séries nées dans le sillage de Nick Carter suffiront à se faire une idée du processus de sérialisation et des effets de sens qu'il dessine ${ }^{27}$ : Bill Cannon, Kriminalkommissar (Allemagne, 1908), Amerikanischen Detectivs John Wilson (Allemagne, 1908-1910) Miss Boston (France, 1910), Jongen Nelson, Amerika's grooste detectives (Pays-Bas, 1919), Jack Dollar (France, 1922), Nick Pratt, Amerikas Meisterdetectiv (All., 1922) Todd Marvel, détective milliardaire (France, 1923) Americké detektivní povidky; Dobrodruzstvi Craiga Kennedyho (Tchécoslovaquie, 1924), Jim Joyce o "az» dos detectives americanos (Portugal, 192 ?). Toutes ces séries de fascicules soulignent dès leur titre le lien avec le modèle américain, ou tout au moins avec le modèle anglo-saxon ${ }^{28}$. De même, derrière Buffalo Bill, c'est l'Amérique de la frontier qui s'impose partout. Dans les deux cas, il s'agit bien de jouer avec la fascination pour les États-Unis, qui est l'un des éléments d'attraction produisant de la nouveauté pour les lecteurs. Autrement dit, quand bien même ils retrouvent en réalité des imaginaires déjà présents en Europe, Buffalo Bill et Nick Carter incarnent chacun un genre américain, celui du récit de l'Ouest pour le premier, et celui du récit d'aventures policières pour le second.

12 Par rapport à Buffalo Bill, Nick Carter importe en outre un imaginaire de la ville moderne américaine ${ }^{29}$. Les récits se situent dans des grandes cités, peu décrites, mais suffisamment caractérisées pour nourrir les fantasmes du lecteur ${ }^{30}$. Cette impression de modernité passe peut-être plus encore par les couvertures que par le texte lui-même. On y découvre des immeubles, des voitures, des salles de cinéma... autant d'images peu présentes encore dans les gravures des feuilletons à l'époque, autant de traits frappants sur les couvertures en couleurs. En France, cette modernité clinquante semble avoir marqué les contemporains, puisque les ersatz de Nick Carter développent fréquemment un imaginaire de la modernité technologique, à l'instar de Tip Walter (1911-1912) et de Miss Boston (1910), qui recourent volontiers à la TSF, au téléphone et aux aéroplanes, preuve que ce trait est en partie une construction de la réception française ${ }^{31}$. Mais c'est aussi le cas dans les autres pays européens où, dans le sillage de Nick Carter, se multiplient les séries mettant en valeur la modernité des détectives anglo-saxons ${ }^{32}$, à travers un certain nombre de caractéristiques significatives. Les personnages sont d'abord associés à un imaginaire cosmopolite (retrouvant le caractère international de la diffusion). Ce sont des milliardaires ${ }^{33}$ et des «détectives mondiaux " 
(« weltdetectiv $)^{34}$, et à l'époque un tel cosmopolitisme renvoie à la modernité technique du voyageur fortuné. C'est ce que montre leur usage immodéré de voitures, aéroplanes, et appareils de communication qui tiennent une place ostentatoire dans les œuvres. Cette modernité s'affirme aussi dans le rythme des récits. Loin du modèle cérébral des nouvelles holmesiennes, les Nick Carter s'imposent comme des romans d'aventures délestés des longueurs du feuilleton. Retrouvant le stéréotype de l'homme d'action américain et l'idée d'une modernité faite de vitesse, ce rythme peut aussi être perçu comme un trait d'américanitée ${ }^{35}$. La même densité peut se lire dans les aventures de Buffalo Bill. Ces romans brefs à l'intrigue sans temps mort correspondent aux stéréotypes de l'Amérique, Nation se transformant rapidement, rivalisant avec l'Europe au point d'être prête à la dominer.

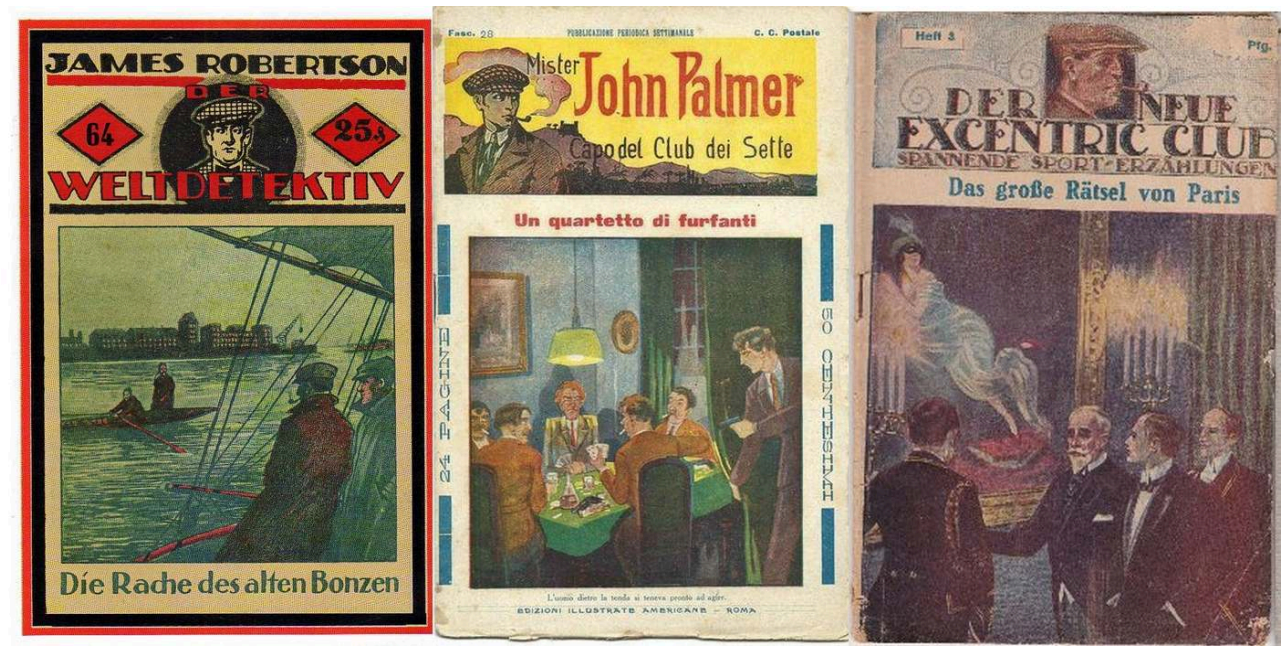

Image 6 à 9 : Weltdetectiv, milliardaires et excentric clubs : l'imaginaire moderniste des séries de fascicules.

\section{Spectacle et transmédialité}

Mais si ces récits ont pu apparaître comme modernes, c'est aussi parce qu'ils se sont inscrits très vite dans une culture transmédiatique. Le lien avec le spectacle et la publicité associait déjà les personnages avec un imaginaire médiatique. La chose est évidente pour Buffalo Bill, forain et homme de spectacle, qui avait consciemment construit sa propre célébrité en explorant tous les médias et les modes d'expression littérature, presse, théâtre, wild west shows, affiches, photographies. Mais cette logique publicitaire apparait également chez Nick Carter, dont le texte et le paratexte ne cessent de vanter la célébrité36, et dont le portrait est généralement reproduit en couverture. Il s'agit bien, dans ce cas, d'orchestrer la notoriété du personnage, et de faire de Nick Carter l'équivalent, pour le récit de détective, de ce qu'est Buffalo Bill pour le western. La présentation même des fascicules en fait des livres-affiches, avec leurs couvertures coloriées destinées à attirer l'œil du spectateur comme le font les affiches placardées sur les murs $^{37}$. On en voudrait encore pour preuve la propension des épigones à orchestrer eux aussi leur célébrité, transformant leurs couvertures en autant d'affiches tapageuses $^{38}$.

14 Mais cette transmédialité va rapidement se concrétiser dans tout un ensemble d'adaptations. Avec leurs récits brefs synthétisés par des couvertures-affiches, les fascicules d'Eichler s'y prêtent évidemment tout particulièrement. Ce sera le cas en 
particulier de Nick Carter, repris au cinéma dès 1908 par Victorin Jasset et au théâtre en 1910 par Alexandre Bisson et Guillaume Livet, et donnant lieu à toute une série de parodies dont la plus fameuse prendra la forme de films autour du personnage de Nick Winter, à partir de 1912. Mais on peut citer également le cas des adaptations de Nat Pinkerton (Danemark, 1909, puis France, 1913, et Allemagne, 1921) ${ }^{39}$. Quant à Buffalo Bill, sans doute trop célèbre pour être incarné de son vivant par un acteur, il verra son univers décliné dans une vague de films de l'Ouest, dont ceux, fameux, de Jean Durand. Dans plusieurs pays, cette dynamique transmédiatique s'est concrétisée dans des séries de fascicules dont les héros-détectives sont empruntés au cinéma, à l'instar de Nick Winter (Espagne, 1910, repris de la série de films français), Maciste (Pays-Bas, 1919, inspiré par le héros de films italiens), Harry Piel (Allemagne, 1920, d'après l'acteur Allemand) ou Elmo Lincoln (Pologne, 1924, d'après l'acteur américain).

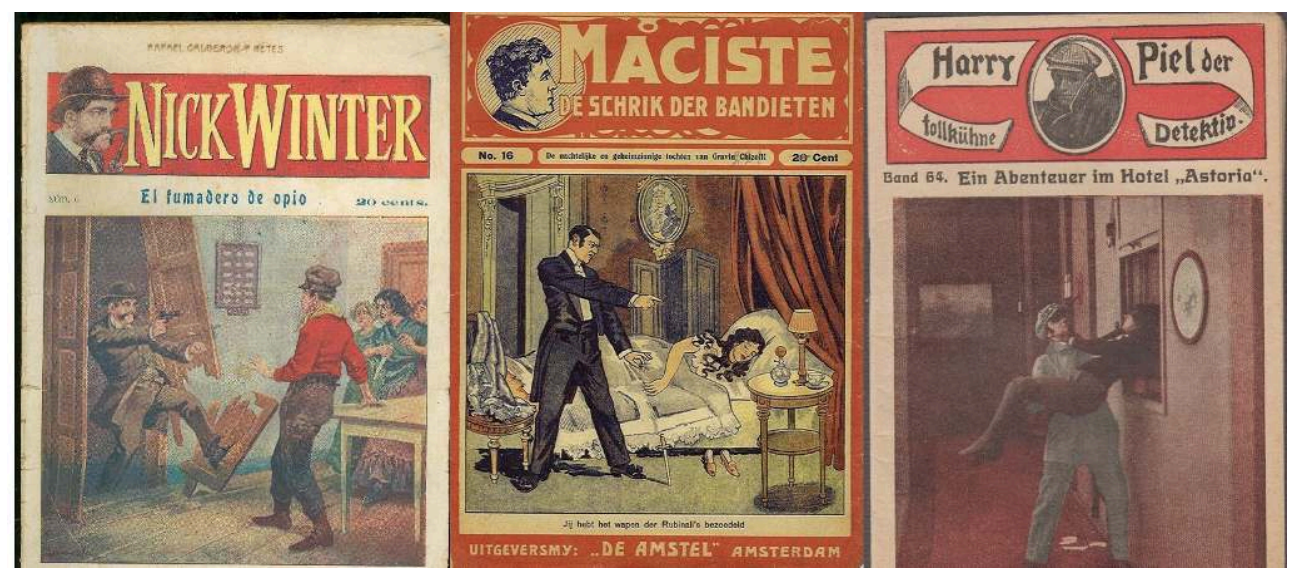

Images 10 à 12 : Les séries de détectives Nick Winter, Maciste et Harry Piel, expressions d'un imaginaire médiatique

15 Le cas de l'Espagne montre plus particulièrement comment cette dimension médiatique peut se ressentir aussi dans des marchés périphériques. Dans ce pays offrant avant la Première Guerre mondiale un accès réduit aux salles de cinéma, ce sont les théâtres qui investissent ces récits, en proposant toute une série de pièces improvisant de nouvelles aventures de Nick Carter, Sherlock Holmes, Raffles, Fantômas, et imaginant des rencontres inédites entre les uns et les autres ${ }^{40}$, dans des intrigues souvent chantées qui conservent des traits des zarzuelas populaires. Or, ces personnages sont aussi ceux qui triomphent au cinéma. Et c'est sans doute l'attrait de ce nouveau média, relayé par les fascicules et les éditions populaires, qui explique les reprises au théâtre, comme pour accéder malgré tout à un ersatz de cette modernité médiatique.

A travers les deux séries lancées par Eichler et leurs imitations européennes, on voit donc se dessiner un ensemble architextuel cohérent. Il associe deux architextes génériques - le récit de détective et le récit de l'Ouest, un univers culturel, celui de l'Amérique, et un imaginaire médiatique qui lui est associé, celui du spectacle, de la publicité et de la modernité. Tous ces traits s'articulent autour d'un type de support, le fascicule, qui associe formats, intrigues, imaginaires et logiques industrielles pour dessiner une série culturelle cohérente. Il y a en effet homogénéité de la série culturelle

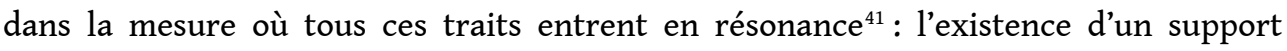
importé d'Amérique, le dime novel, les techniques de diffusion internationales, la publicité ou la circulation transmédiatique des imaginaires renforcent cet effet de cosmopolitisme des intrigues internationales insistant sur l'américanité et le 
modernisme. Fascicules, théâtre et cinéma policiers ou de l'Ouest peuvent alors être condamnés dans les mêmes termes, parce qu'ils sont soupçonnés de participer d'une même culture associée à la modernité, populaire et infantile, immorale et pousse-aucrime. Le discours contemporain ajoute un dernier trait, celui de l'origine étrangère des imaginaires - visant à la fois l'importateur allemand, quand on veut tancer l'immoralité des productions ${ }^{42}$, et l'origine américaine, quand il s'agit de renvoyer à un imaginaire et à une culture vulgaire, mercantile et violente.

Cette dynamique médiatique mondiale apparait nettement dans les réactions des contemporains en Norvège, lors de la panique morale des années 1908-1909 déclenchée par l'arrivée de ces fascicules ${ }^{43}$. Ce qui est inlassablement évoqué par la presse, c'est leur circulation internationale et l'impossibilité de les stopper aux frontières : venus du Danemark, qui les reçoit lui-même d'Allemagne, importés des États-Unis, ces fascicules semblent échapper à tout contrôle, et sont perçus à travers un vocabulaire de "l'invasion étrangère » et de "l'épidémie ». Surtout, lors même qu'ils sont distribués en quantité relativement modeste en Norvège (entre 3 et 8000 exemplaires par numéro, pour un tirage de 20 à 25000 exemplaires par semaine pour l'ensemble des collections de fascicules ${ }^{44}$ ), ils sont associés aux tirages considérables des voisins allemands ${ }^{45}$. On voit combien l'inquiétude est accrue par le caractère international, nourrie en particulier par l'écho médiatique des réactions des pays voisins. En ce sens, le phénomène représenté par ces publication est aussi mondialisé en cela que les médias européens se nourrissent les unes des autres, ce qui a pour effet d'accroître en particulier l'inquiétude des pays culturellement périphériques (Norvège, Bohème) à travers le prisme des réactions outrées des pays centraux (France, Allemagne).

\section{Séduction des imaginaires et mutations culturelles}

Reste à comprendre les conditions du succès des récits publiés par Eichler. Pourquoi se sont-ils imposés à si grande échelle et si vite alors qu'ils ont immédiatement souffert d'un dédain critique, et ont été condamnés par les autorités morales ? Pourquoi ont-ils si rapidement été imités? Pourquoi néanmoins ces imaginaires ont-ils pu s'étioler et disparaitre après quelques années d'un succès fulgurant? Pour le comprendre, il convient d'examiner les discours des contemporains. Lorsque les articles évoquent les fascicules d'Eichler, ils mettent l'accent sur le rôle joué par ce nouveau support dans l'association entre un type de textes (les récits criminels ou d'aventures dans l'Ouest) et un lectorat (les jeunes lecteurs). Ainsi serait-ce moins un nouveau genre de récits qui aurait fait souche qu'un nouveau genre de publications - c'est-à-dire non seulement ce support américain (pas si original en Europe, on l'a vu), mais son articulation avec des univers et des intrigues avec lesquels il entre en résonance. Et de fait, on l'a vu, ce qu'achète et importe Eichler, c'est autant une série de romans qu'un type de produit saisi dans sa globalité, avec un format, une mise en page, une maquette de couverture, une illustration et un bandeau, et même une quatrième de couverture, étonnamment similaire entre les différents pays ${ }^{46}$, tous importés des États-Unis, et diffusés synchroniquement dans toute l'Europe. Il est clair qu'il y a une volonté d'importer un objet sémiotique global, produisant un architexte dont la signification est perçue, de façon indissociable, dans l'unité de la série textuelle et de la série éditoriale.

On voit que la circulation des œuvres dépasse largement ici celle des textes et de leur traduction. Habituellement, l'importation d'un récit suppose de l'adapter au système 
éditorial d'accueil : supports, collections et unités éditoriales différent suivant les pays, produisant leurs propres cohérences discursives. Et les ajustements médiatiques, en entraînant une négociation entre les discours sériels d'origine et ceux de la culture d'accueil, participent de la reconfiguration du texte à partir des imaginaires sériels du nouveau pays. Et dans une certaine mesure, l'arrivée du dime novel s'est traduite par une négociation avec le système éditorial de l'époque (celui des kiosques, marchands de tabac, colporteurs, etc.). C'est ce qui explique le choc produit par ces collections, qui affichent leur exotisme à travers la matérialité même du support, tout en investissant des réseaux existants. Il faut donc comprendre l'événement que représente leur arrivée en Europe comme un événement médiatique, dont la signification se situe au croisement de la matérialité du support et des imaginaires fictionnels sérialisés.

Cette logique globale produit une cohérence, assurée dans un même mouvement par le personnage (au niveau de la diégèse) et par l'éditeur (au niveau de l'énonciation), qui marginalisent le garant traditionnel du texte, l'auteur. De fait, le poids de l'éditeur est renforcé par l'effacement de l'auteur. Les aventures de Buffalo Bill sont écrites «par l'auteur de Buffalo Bill»; celles de Nick Carter, "par l'auteur de Nick Carter », sans autre précision que cette vague formule tautologique ${ }^{47}$. Autrement dit, elles n'ont pas d'auteur, parce que l'autorité véritable assurant l'unité de signification, la figure d'auctorialité, c'est l'éditeur (Street and Smith aux États-Unis, Eichler et ses épigones en Europe). C'est en ce sens que se détermine un pacte de lecture éditorial. Confirmant ce déplacement de la fonction-auteur, c'est généralement Eichler qu'on attaque quand il s'agit de chercher un responsable à l'origine des œuvres.

21 Mais si la série éditoriale assure l'unité d'énonciation, elle est à son tour reformulée en auctorialité diégétique, puisque - et c'est là une nouveauté très forte, première occurrence d'un processus de sérialisation diégétique appelé à devenir central au XXe siècle - les personnages reviennent d'un épisode à l'autre, assurant l'unité entre la collection éditoriale et l'unité transfictionnelle. La célébrité orchestrée de Nick Carter et de Buffalo Bill, avec leur nom en grosses lettres, leur portrait identifiable et le paratexte insistant sur leur notoriété, en faisait d'autres figures d'auctorialité. Certaines quatrièmes de couverture présentaient Buffalo Bill et Nick Carter comme les auteurs des romans contant leurs exploits ${ }^{48}$. Pour ce dernier, il reste au moins une trace de cette confusion, une série de lettres, restituées par un journal américain de 1917, adressées par différents lecteurs, français, belges, espagnols, russes, à «l'estimé Nick Carter $»^{49}$. Et Eichler lui-même soulignait cette confusion comme gage de son succès ${ }^{50}$. En déplaçant l'auctorialité à la fois du côté de l'éditeur et du personnage, l'intérêt de la série se déplace doublement hors du texte. L'Allemand Eichler est la source énonciative qui porte la logique médiatique, et Nick Carter et Buffalo Bill celles qui enracinent la référence à une Amérique réelle.

22 C'est là que réside la révolution d'Eichler, dans l'invention d'une série médiatique, d'une série éditoriale et d'une série diégétique comme réalités indissociables, dont l'articulation produit une nouveauté radicale indépendamment de la nouveauté des récits eux-mêmes. La diffusion massive, dont l'effet est rapidement redoublé par l'apparition de concurrents démarquant le support du fascicule, produit une saturation de l'espace médiatique. Cet effet de masse est accru par la concordance entre les supports standardisés et les contenus sérialisés : les imitateurs reprennent à la fois un format (le fascicule), et des genres de textes (le récit de détective et le western), produisant un effet synchronique entre série médiatique et série générique. Enfin, à un 
niveau supérieur, la logique de publicité et de célébrité relayée par la circulation transmédiatique convertit le phénomène médiatique en phénomène culturel. Tous ces éléments se combinent évidemment pour expliquer l'effet produit par l'arrivée des fascicules Eichler. C'est ce dont témoigne l'étonnante place prise par les références aux deux séries dans les médias, par exemple en France, où l'on en parle sans cesse entre 1908 et 1911, témoignant qu'il s'agissait d'un phénomène culturel majeur - quand bien même ce serait parce qu'il est fustigé par les catholiques et les instances éducatives ${ }^{51}$. Mais c'est le cas aussi par exemple en Allemagne, en Tchécoslovaquie, en Norvège ou en Suède, où la série a produit à chaque fois de véritables paniques morales ${ }^{52}$. En particulier, les commentateurs ont bien compris le tournant industriel (et donc pour eux anti-culturel) de ces productions que manifestent la masse de fascicules et leur développement rapide, on l'a vu.

Or, cette logique industrielle qui impose des supports très bon marché, entraîne de fait une mutation de la communication, qui explique l'accusation d'immoralité et de pornographie qui frappe si souvent les publications Eichler. En effet, celle-ci porte moins sur leur contenu (le Grand Guignol se développe dans ces années, et la violence des publications Eichler est sans commune moindre que celles des feuilletons de l'époque $\left.{ }^{53}\right)$ qu'à la transformation des modes de consommation qu'induit le support. Car le véritable reproche qui est fait à ces fascicules très bon marché, c'est de rendre accessibles des récits de crime aux jeunes lecteurs, qui peuvent les acquérir sans difficultés, loin du contrôle des parents ou des instituteurs ${ }^{54}$. Qu'on évoque le caractère pernicieux des textes ou leur naïveté, dans tous les cas, ce qui importe, c'est l'existence d'un support que son prix rend accessible aux jeunes lecteurs - ce qui est, de fait, une nouveauté à l'époque, puisqu'auparavant, le marché du roman pour la jeunesse était dominé par des ouvrages beaucoup plus coûteux. La nocivité de ces textes ne tient pas tant à leur contenu (ils sont moins violents que ceux du capitaine Danrit ou de Louis Boussenard, leurs contemporains), qu'aux possibilités d'émancipation que représente la lecture buissonnière, loin du contrôle des parents. C'est pourquoi les récits de lecteurs poussés au crime par ces publications sont toujours des anecdotes de lecteurs seuls, ou entre camarades ${ }^{55}$. C'est ce contexte de consommation qui représente sans doute le véritable danger - mais aussi la véritable modernité de ces textes. Lire hors du regard des parents et des problématiques de transmission, c'est déjà transgresser l'autorité. Autrement dit, ce qui inquiète face à ces objets, c'est qu'ils manifestent les transformations des mœurs qui affectent les grandes capitales européennes et les villes des pays les plus développés : ils correspondent à de nouvelles formes de loisirs et de divertissement, à de nouveaux modes de consommation, dominés par le plaisir plus que par la morale (d'où l'accusation de pornographie et la peur du vice). Et le fait qu'ils sont tout particulièrement consommés par les jeunes lecteurs (c'est-à-dire par ceux qui seront les plus touchés par cette nouvelle culture urbaine) inquiète davantage encore, puisque dans l'enfant se cristallise la question de la transmission et de l'avenir du modèle social. En ce sens, ces fascicules manifestent aussi une mutation culturelle plus large, ou en tout cas le sentiment d'une mutation en marche, quand bien même, comme dans les pays moins développés, cette mutation serait essentiellement fantasmée, à travers des discours médiatiques colportant les craintes des voisins plus développés. 


\section{Nouveaux supports et nouvelles logiques du récit} nouveaux décors urbains et de nouveaux personnages diégétise une révolution médiatique qui les impose. Cela ne signifie pas qu'il n'y a pas de transformations fondamentales des formes littéraires. Mais celles-ci découlent des logiques médiatiques bien plus que d'une spécificité à rapporter à la culture américaine. Nous ne reviendrons pas sur ces questions que nous avons eu l'occasion d'aborder ailleurs ${ }^{56}$, et nous contenterons d'en résumer quelques traits. Ainsi, le faible nombre de pages offertes par ce support impose des récits resserrés qui inversent totalement les logiques du feuilleton, produisant un effet de rapidité qu'on a eu tôt fait d'associer aux intriques américaines. L'apparition de supports de diffusion comme le fascicule et la collection populaire au début du XXe siècle se traduit par le déclin rapide du feuilleton et des formes qui lui sont associées comme paradigme médiatique dominant de la culture populaire en France. Elle s'accompagne d'un renouvellement complet du système générique et plus largement des structures architextuelles qui lui sont associées, et d'un changement plus fondamental de la fonction du genre dans la communication littéraire. Enfin, l'usage de la couverture illustrée et des formats standardisés organisés en collections va se pérenniser dans le premier XXe siècle pour déterminer un nouveau pacte de lecture, faisant refluer l'auteur au profit du genre et de la collection, et invitant à saisir la spécificité de l'œuvre au sein de ce genre à partir des informations synthétiques de la couverture, lesquelles déterminent largement l'horizon d'attente. Tous ces processus se retrouvent dans ces brefs récits à personnages récurrents marqués par une forte identité générique.

Ainsi, ces mutations majeures découlent davantage de la révolution représentée par le support que de celle engagée par les textes et par leurs imaginaires. De fait, dans bien des pays, les récits de détectives ou de l'ouest ont été absorbés par d'autres formes sérielles. Le weltdetectiv allemand qui combattait le crime un peu partout dans le monde s'est confondu avec les aventuriers impérialistes que l'Allemagne affectionnait toujours davantage dans les années 1920 et 1930. De même, en France, les récits de l'Ouest ontils été absorbés dans les collections populaires de romans d'aventures, et les héros coloniaux des fascicules traversent l'Amérique comme une simple étape dans leur voyage autour du monde. Plus généralement, dès les années 1920, l'influence des récits de détectives cède un peu partout le pas à celle des romans à énigme et des shokers britanniques puis, dans les années 1930, aux récits de gangsters à l'américaine. Mais ce retour à des modèles nationaux et à leurs héritages n'a en revanche pas remis en cause les mutations médiatiques qui se sont produites. Les œuvres ont définitivement adopté les formats courts et les esthétiques qu'ils induisaient; elles sont restées liées aux modèles des collections, et ont pris acte des mutations génériques qu'a imposées le changement de paradigme médiatique. C'est là que résidait la véritable révolution apportée par ces supports, et celle-ci a achevé de s'imposer dans l'entre-deux-guerres. Le récit criminel peut bien s'éloigner - un temps - des décors américains et des détectives, il a adopté les nouvelles structures du roman policier; il a contribué à l'abandon du modèle social et collectif au ton mélodramatique, génériquement hybride et narrativement ramifié du récit criminel du XIXe siècle, au profit du modèle resserré et plus homogène qu'ont imposé les formats $d u$ fascicule ${ }^{57}$. Quant aux récits d'aventures, ils ne se situent peut-être plus dans l'Ouest, mais ils ont adopté le principe

Belphégor, 18-1 | 2020 
d'une intrigue resserrée autour d'un événement unique, plus proche de celle des Buffalo Bill d'Eichler que des romans-feuilletons et de leurs récits de voyage, de chasse et de tour du monde. Surtout, à la fin des années 1920, on a accepté le principe d'une littérature de divertissement pour la jeunesse, tentant tout au plus de la contrôler et de la canaliser. De nouvelles formes de consommation se sont imposées, et avec elles, de nouvelles logiques esthétiques.

\section{Une révolution médiatique européenne}

Si ces séries américaines ont bouleversé les imaginaires européens, c'est moins grâce aux thèmes du détective et du cowboy que par la rupture structurelle entraînée par l'arrivée du format même du dime novel. Les contemporains l'avaient intuitivement compris en attaquant la vulgarité des couvertures ou les modes de lecture induits par les supports. Ils avaient également compris que la révolution était autant allemande qu'américaine : c'est en imposant brutalement de nouveaux supports grâce à l'efficacité de logiques industrielles révolutionnaires qu'Eichler a contribué à bouleverser les pratiques de consommation et bientôt les manières d'écrire. Si dans les pays à la marge de la mondialisation médiatique de telles pratiques ont été en partie absorbées par les vieilles logiques $\mathrm{du}$ colportage et de l'édition populaire traditionnelle (Tchécoslovaquie), dans les Nations plus au cœur de la modernité médiatique (France, Allemagne) et dans les grandes villes de nombreux pays (Italie, Pays-Bas, Belgique, Pays Nordiques), elles ont engagé l'édition populaire sur la voie de ce fordisme littéraire qui allait caractériser le XXe siècle. Mais l'évocation de l'Allemagne et de l'Amérique, comme celle des nouvelles logiques de production ou de distribution qui sont associées à ces supports, montrent que même dans les espaces périphériques (Tchécoslovaquie, Hongrie $^{58}$ ), le phénomène est perçu à travers ces mêmes caractéristiques - celles d'une mondialisation des imaginaires et des logiques industrielles qui les portent. Cela signifie que, même dans des espaces plus à la marge des mouvements de mondialisation, où ils ont été limités dans leur diffusion, souvent expérimentés de manière plus tardive, et absorbés en particulier par des logiques plus anciennes, comme le colportage, ces phénomènes ont été malgré tout perçus comme l'expression d'une mutation culturelle majeure, associée à une marchandisation et à une industrialisation de la littérature, contribuant, dans ces espaces aussi, à la mondialisation des imaginaires. S'ils ont pu être là aussi perçus comme des événements, c'est parce que la presse n'a cessé de commenter ce qui se passait ailleurs, en France ou en Allemagne (comme l'ont fait la Norvège ou le Danemark), participant, au moins par les commentaires, à cet événement médiatique et culturel globalisé.

Ce que nous avons voulu plus généralement montrer, c'est que l'on a tort d'étudier les transformations de la littérature populaire à partir des seuls textes ou mêmes des architextes (ceux des genres par exemple) indépendamment de leurs conditions de production et de consommation. Parfois, ce sont moins les textes que les contextes de communication qui altèrent les imaginaires et les formes. Ou pour le dire autrement, on ne peut pas comprendre l'incidence des textes sur les transformations des imaginaires si l'on oublie que, comme tout acte de langage, ils sont fondamentalement produits par leur situation culturelle et médiatique de communication. Les textes n'existent qu'à travers leur matérialisation dans un cadre social via des objets qui s'organisent dans l'espace médiatique, économique et interdiscursif. Ce qu'entendaient 
les contemporains d'Eichler, ce n'était pas seulement le bruit du revolver du détective ou celui du six coups du pionnier, mais aussi celui des pages d'un nouveau type de support bon marché aux couvertures criardes et des valeurs qui lui étaient associées, qui allaient contribuer à faire basculer la littérature populaire européenne dans le XXe siècle.

\section{NOTES}

1. Cette réflexion prolonge en les étendant à l'Europe les recherches que nous avons déjà entreprises dans « 'L'Amérique envahit la France' - Les épigones de Nick Carter et la redéfinition de l'imaginaire criminel urbain à la Belle Époque ", in Catherine Nesci, American Mysterymania, Media19, 2018 http://www.medias19.org/index.php?id=23828.

2. Michael Denning, Mechanic Accents; Dime Novels and Working-Class Culture in America, Londres, New York, Verso, 1987.

3. Date donnée par Quentin Reynolds dans The Fiction Factory, or From Pulp Row to Quality Street, New York, Random House, 1955.

4. Alberto Menarini et Franco Cristofori, Eroi del racconto popolare; Prima del fumetto, Bologne, Edison, 1986.

5. Christian Huck, "American Dime Novels on the German Market: the Role of Gatekeepers ", in Christian Huck, Stefan Bauernschmidt (dir.), Travelling Goods, Travelling Moods; Varieties of Cultural Appropriation (1850-1950), Chicago, University of Chicago Press, 2012. Georg Jäger donne quant à lui un tirage (déjà considérable) de 45000 exemplaires («Der Kampf gegen Schmutz und Schund ; Die Reaktion der Gebildeten auf die Unterhaltungsindustrie », Archiv für Geschichte des buchwesens, 31, 1988).

6. Rimmer Sterk et Jim Conkright, The Continental Dime Novel, s. l., JCRS, 2006. Dans l'interview que le journal américain Printer's Ink accorde à Eichler en 1906, celui-ci évoque également la Suède.

7. Par exemple dans The Minneapolis Journal, 17 juillet 1907 (« Nick Carter in German », parodie qui témoigne du bruit médiatique que produit en Amérique l'annonce du succès de la série en Allemagne), dans Printer's Ink, 1906 (« Nick Carter in Europe »), dans The Literary Digest, vol. 38, 1908 («Emigration of Dime Novels ») ou dans The Atlanta Constitution, 7 novembre 1909 («Danish people turning to American literature »), pour ne prendre que quatre exemples.

8. Rimmer Sterk évoque, pour la série Buffalo Bill, une ou plusieurs séries belges (1924), croates (1928), estoniennes (1912), finlandaises (1924), norvégiennes (1909), ottomanes (1926), russes (1909 ?), espagnoles (1910?) et suédoises (1906). Quant à la série des Nick Carter, elle est publiée, par Eichler ou d'autres, en Belgique (1925), Bohème (1908), Croatie (1906), Danemark (1908), Estonie (1908), Finlande (1904), Hongrie (1906), Italie (1908), Pays-Bas (1908), Norvège (1915), Empire Ottoman (1909), Pologne (1907), Portugal (1910), Russie (1907), Espagne (1910) et Suède (1902 et 1906). Rimmer Sterk et Jim Conkright, op. cit.

9. Si Printer's Ink écrit plaisamment que seules la Russie et la Turquie sont épargnées en Europe, il se trompe, puisque l'une et l'autre diffuseront les aventures de Nick Carter et de Buffalo Bill (op. cit.).

10. C'est ce qui explique qu'en Italie, l'éditeur Nerbini pourra relancer dès 1919 la série des Nick Carter après la faillite d'Eichler, simplement parce que le format était très proche des fascicules 
qu'il distribuait depuis des années déjà (voir Fausto Colombo, La Cultura sottile; Media e industria culturale in Italia dall'ottcento agli anni novanta, Milan, Bompiani, 1998).

11. Les cartes postales des devantures de kiosques à journaux et de merceries offrent un témoignage de la manière dont ces productions s'affichaient. Voir image 5.

12. En Suède, la panique morale qu'a engendré l'apparition des romans en fascicules en 1908-1909 s'est traduite par l'attaque des «marchands de tabac», qui en étaient les principaux distributeurs. Ulf Boëthius, "Ungdomar, medier och moraliska paniker », in Johan Fornäs, Ulf Boëthius och Bo Reimer (éd.), Ungdomar i skilda sfärer, Stockholm, Brutus Östlings Bokförlag Symposion AB , 1994.

13. Sur la vente de ce type de produits en colportage, voir par exemple Ronald A. Fullerton, «Toward a Commercial Popular Culture in Germany: The Development of Pamphlet Fiction, 1871-1914 », Journal of Social History, Vol. 12, No. 4 (été 1979) ; pour le Danemark, voir Per Hougaard, «Om knaldromaner, føljetoner og kolportage-litteratur i Danmark (I) », Magasin fra Det kongelige Bibliothek, 11 (1996:3); pour la Tchécoslovaquie, Michal Jareš, Cliftonovo Století, mémoire de thèse sous la direction de Pavel Janousek, Université Palacky, Olomouc, 2018.

14. Sur le caractère central ou périphérique des pays européens, voir Franco Moretti, Atlas du roman européen, Paris, Seuil, « La couleur des idées », 2000.

15. On trouve une sélection de ces « cliftonky » sur le site Digitální archiv populární literatury, http://dapl.ucl.cas.cz/ostatni-povidkove-serie (consulté le 9 janvier 2020).

16. On en trouve un témoignage direct dans Hermann Leopold Köster, qui constate que si la littérature ordurière (Schundliteratur) a toujours existé dans l'indifférence générale, « les choses ont changé au début du 20e siècle. La grande entreprise moderne a pris le contrôle de ce type de productions. Le mal a commencé en Amérique. Les premières brochures contenaient les mémoires présumés de Buffalo Bill et les actions du détective Nick Carter. Ce nouveau type de littérature ordurière reposait sur deux innovations : une distribution organisée autour de gros tirages et le principe de la "série" [...] Une organisation commerciale bien développée veillait à ce que tout le pays soit inondé de ces livrets" (notre traduction), Geschichte der deutschen jugendliteratur in monographien, Hambourg, Janssen, 1906-1908. Cette rupture représentée par l'industrialisation de pratiques ancienne est analysée pour l'Allemagne par Patrick Major, "'Smut and Trash': Germany's Culture Wars Against Pulp Fiction », in Karl Christian Führer et Corey Ross, Mass media, culture, and society in twentieth-century Germany, New York, PalgraveMacmillan, 2006; pour la Tchécoslovaquie, voir Michal Jareš, op. cit.

17. On trouve également ces chiffres dans un article du Nieuwsblad voor den boekhandel. 78, 1-52 (1911).

18. Ces deux séries sont publiées sous la marque de "La Nouvelle populaire », mais c'est bien Eichler qui est derrière cette marque.

19. Par exemple, pour les Pays-Bas et l'Allemagne, l'article hollandais (sans titre) du Nieuwsblad voor den boekhandel. 78, 1-52 (1911); pour l'Allemagne, voir Hermann Leopold Köster, Geschichte der deutschen jugendliteratur in monographien, Hambourg, Janssen, 1906-1908; pour la Suède, Ulf Boethius, op. cit.

20. Dieter Sudhoff et Hans-Dieter Steinmetz, Karl-May-Chronik: 1897-1901, Radebeul, Karl-MayVerlag, 2005.

21. Don Russell, The Lives and Legends of Buffalo Bill, Norman, University of Oklahoma Press, 1979.

22. Robert W. Rydell et Rob Kroes, Buffalo Bill in Bologna; The Americanization of the World, 1869-1922, Chicago et Londres, The University of Chicago Press, 2005.

23. Richard Slotkin, Gunfighter Nation, Norman, University of Oklahoma Press, 1998.

24. Dominique Kalifa, Histoire des détectives privés en France (1832-1942), Paris, Nouveau Monde, 2007.

25. Loïc Artiaga et Matthieu Letourneux, Fantômas, biographie d'un criminel imaginaire, Paris, Les Prairies ordinaires, « Singulières modernités », 2013. 
26. On trouve une liste impressionnante de ces séries (plusieurs centaines, soit des milliers de titres), dans Rimmer Sterk et Jim Conkright, op. cit.

27. Sur la manière dont la sérialisation des imaginaires produit des effets de cohérence, voir Matthieu Letourneux, Fictions à la chaîne, littérature sérielle et culture médiatique, Paris, Seuil, « Poétique », 2017.

28. Nombreux sont en effet les détectives britanniques. Cela s'explique par l'influence de Sherlock Holmes. Reste que le Sherlock Holmes qui est diffusé en fascicules n'est pas celui de Conan Doyle, mais le « Detectiv Sherlock Holmes » apocryphe des Allemands (1907, 230 épisodes), qui circulera dans une vingtaine de pays d'Europe sous différents noms (« Les dossiers secrets du roi des détective», etc.) jusqu'à devenir, en France «Harry Dickson, le Sherlock Holmes américain ", preuve de la confusion entre l'Amérique et l'Angleterre dans les récits de détectives. Ce Sherlock Holmes de fascicules n'est en effet pas un détective déductif, mais un homme d'action calqué sur Nick Carter.

29. Ce décor de ville criminelle explique d'ailleurs que les séries de détectives aient davantage que les récits de l'Ouest suscité les critiques des censeurs. Sur cette question, voir Raphael Rössel, «Das Lesen der Schundkämpfer; Moralpaniken als strategische Deutungen populärer Literatur am Beispiel der Kontroverse um Nick Carter (1906-1913)», ZS; Internationales Archiv für Sozialgeschichte der deutschen Literatur (IASL), 44, 1 (2019).

30. Ce qu'explicite un titre comme "Gentleman detektiven. Stordstads-Mysterier » (Danemark, 1917), mais que l'on rencontre dans bien des récits, qui se situent volontiers dans de grandes cités marquées par leur modernité.

31. Sur cette question d'une modernité américaine et technologique dans les séries de fascicules français, voir Matthieu Letourneux, «La disparition du genre des mystères urbains ", , in Dominique Kalifa et Marie-Ève Thérenty, Les Mystères urbains au XIX ${ }^{e}$ siècle: Circulations, transferts, appropriations, Médias 19, 2015. Publication en ligne sur le site Media 19: http:// www.medias19.org/index.php?id=17039.

32. A l'instar de la série « Pik Linder, Poliziottio moderno » (192 ?).

33. En France, ce sera la série «Todd Marvel, détective milliardaire », 1923, mais les détectives des séries allemandes et italiennes sont souvent également richissimes, à l'instar de « Lord Percy Stuart vom Excentric Club» (1913) et «Neue Excentric Club» (1920) ou de la série italienne « Mister John Palmer, capo del Club dei Sette » (1930).

34. La figure du «weltdetectiv» se développe en Allemagne, mais s'exporte ensuite dans toute l'Europe : on citera les séries « Jack Franklin, der weltdetectiv » (1909) ou « James Robertson, der weltdetektiv» (1921). Dans une relance tardive de "Nick Carter» en Allemagne, celui-ci deviendra également un « Weltdetektiv» (1929).

35. Christian Huck, « travelling Detectives: twofold Mobility in the appropriation of Crime Fiction in Interwar Germany », Transfers, 2, 3, hiver 2012.

36. Dans le paratexte éditorial, on le présente le plus souvent comme «le plus grand détective d'Amérique » et « le plus célèbre détective du monde ».

37. Ce trait est systématiquement souligné par les contemporains, quel que soit le pays.

38. «Dr. Morrison, Verdens største opdager» («Dr Morrison, le plus grand détective du monde », Danemark, 1913), «John Siloch, il piu grande poliziotto del mundo» (Italie, 1909), « Klam Siki, il piu celebre poliziotto dei nostri tempi » (1924).

39. Sur cette circulation, voir Federico Pagello, «Transnational connections in European crime film series (1908-1914) », Journal of European Popular Culture, Volume 5 Number 1.

40. Nick Carter apparaît ainsi dans El Ladrón misterioso (1915), El Espía (1915), Los Mascaras negras (1915), La corte del Rey Octavio (1915), La Mano Gris (1915) ) ou encore El corredor de la muerte (1917) ; et dans la pièce comique John y Thum (1918), il croise Sherlock Holmes, Arsène Lupin et Fantômas. 
41. Alain Carou et Matthieu Letourneux, Cinéma, premiers crimes, Paris Bibliothèques, 2015 et « Entre attraction, narration et culture médiatique, le premier cinéma criminel », 1895, printemps 2015.

42. Dans la France de la « ligne bleue des Vosges », la germanophobie vire à l'obsession. Voir par exemple «Les Barbares », Gil Blas, mardi 4 juillet 1911 ou « Un bon exemple », Gil Blas, 17 août 1912.

43. Nous nous appuyons sur les comptes-rendus du Norsk bokhandlertidende, 29-30 (1908-09).

44. Norsk bokhandlertidende, 18 décembre 1908 et 24 septembre 1909

45. C'est du moins ce que laisse penser le chiffre de 165000 exemplaires qui circule un temps, avant d'être dénoncé comme « fantastique ».

46. , «The best detective stories ever written» devient « les meilleures et les plus captivantes aventures de détective qui existent » et l'amorce publicitaire, «we know, boys, that there is no need of introducing to you Nicholas Carter, the greatest sleuth that ever lived ", devient "il n'est pas un Américain, grand ou petit, qui n'ait entendu parler de Nick Carter ». On le voit Eichler reprend l'ensemble du dispositif médiatique américain, jusqu'au paratexte tapageur.

47. Le nom de Frédéric van Renselaer Dey, l'auteur de la plupart des aventures de Nick Carter n'apparaîtra ainsi dans la presse française que bien des années plus tard, quand on apprendra qu'il est devenu fou (« probablement pour avoir eu l'imprudence de lire ses propres œuvres » écrit de façon peu charitable le journaliste du XIXe siècle le 9 septembre 1913).

48. "Nick Carter raconte ses aventures lui-même "; «et c'est vrai! Buffalo Bill a raconté ses aventures dans le sauvage Far-West !»

49. La lettre du Français est par exemple datée du 14 septembre 1909 et envoyée de Nancy. «Cher M. Carter [...] les seules véritables (je crois fermement qu'elles le sont) aventures qui m'ont captivé sont les vôtres. » (notre traduction). La lettre, ainsi que d'autres, est reproduite dans The Soil, vol. $1, n^{\circ} 2$ et 3, janvier et mars 1917.

50. Dans Printer's Ink (op. cit.), Alwin Eichler affirme: «de jeunes lectrices devenues des admiratrices de Buffalo Bill et de ses exploits écrivent constamment pour demander une photographie dédicacée de lui. Un jeune homme a écrit récemment d'une petite petite ville d'Allemagne pour dire qu'il possédait des dons de détective et qu'il aimerait rencontrer Nick Carter pour qu'il l'emploie dans ses extraordinaires enquêtes criminelles » (notre traduction).

51. Nous avons retrouvé une trentaine de textes évoquant les méfaits des publications Eichler auprès des jeunes lecteurs dans la presse française. Jean-Yves Mollier a évoqué ces paniques morales dans La mise au pas des écrivains. L'impossible mission de l'abbé Bethléem au xx siècle, Paris, Fayard, 2014.

52. Pour les paniques morales en Suède, on lira Ulf Jonas Björk, "The Dangerous Prairies of Texas: The Western Dime Novel in Sweden, 1900-1908 ", Swedish-American Historical Quarterly, 55, 2004, et Ulf Boëthius (op. cit.). Pour la Norvège, voir Norsk bokhandlertidende, op. cit. Pour l'Allemagne, Raphael Rössel, «Das Lesen der Schundkämpfe », op. cit. Pour la Tchécoslovaquie, voir Michal Jares, op. cit. Tous soulignent la panique produite par la nouveauté du support, parce que les modes de diffusion qu'il invente le rendent accessible au lecteur, et donc dangereux.

53. Jules Lermina se moque d'ailleurs de ce "thème tout fait " du caractère criminogène des romans de Nick Carter en rappelant que Mandrin et Cartouche étaient nés bien avant l'invention du journal moderne et que les contes de veillées multipliaient les récits sanguinaires. « Dites que c'est bête: bon! quoique vous ne soyez pas fichus d'en faire autant! Mais ne répétez pas cette sottise que nos bandits trouvent en les romans actuels des incitations au crime. C'est faux!» (L'Aurore, 6 août 1910). On trouve une ironie similaire à l'égard de ce prétendu caractère criminogène des fascicules dans le texte hongrois de Schöpflin Aladár, « Nick Carter ; Beszélgetés egy szép asszonnyal », Nyugat, 1908, 24.

54. C'est ce que conte par exemple un proviseur de lycée dans une lettre adressée à L'Echo d'Alger en 1912 ( «les tout petits se cotisent pour acheter des récits de voyage, de ces livres à dix 
centimes aux couvertures bariolées, contenant les exploits d'un Arsène Lupin ou d'un Nick Carter» (10 septembre 1912). Quant à Nieuwsblad voor den boekhandel (op. cit.), il évoque « un enseignant du primaire [qui] a collecté une collection qui ne peut pas être transportée dans une brouette [...] et une table couverte de piles de fascicules confisqués à ses élèves par un autre enseignant » (notre traduction). On voit que la question de la quantité industrielle renforce l'idée du péril.

55. C'est par exemple ce que remarque la Chronique suisse, 1910 : « ce sont surtout les enfants qui achètent cette littérature ; ils la dévorent ouvertement ou en cachette ». Dans L'Aurore (1912), on remarque que «comme ces brochures sont d'un achat facile, même pour les modestes bourses, comme on les trouve dans toutes les gares, dans tous les kiosques, dans les ballots de tous les marchands ambulants, la jeunesse de tous les milieux s'en nourrit et s'en repait ». Ici encore, c'est la diffusion qui produit un péril pour la jeunesse. Par métonymie, on évoque également les pratiques buissonnières des lecteurs de Buffalo Bill : « On a vu des jeunes garçons s'échapper de la maison paternelle pour se rendre dans un coin solitaire entouré d'arbres, et là, tatoués avec de l'encre, des plumes de poule dans les cheveux, ils brandissaient des arcs et des flèches et dansaient, autour d'un feu, à la façon des Peaux-Rouges, une danse guerrière " (Roman revues, "Les Ravages des magazines ", juillet 1908). On renvoie moins à un genre qu'à un type de récits violents publiés sur des supports propres à être consommés par la jeunesse. C'est aussi à la modernité du support qu'Hermann L. Köster impute les dangers de ces lectures (Geschichte der deutschen jugendliteratur in monographien, op. cit.). En Hongrie on souligne que les enfants n'écoutent plus les leçons de leur enseignant, occupés qu'ils sont à rêver sur ces récits qu'on peut acheter n'importe où pour quelques pièces de monnaie (F. P., «Hands off! ", Új idók, 1908 2) Enfin, en Tchécoslovaquie, on affirme que « les enfants lisent ces fascicules à la maison, à l'école, derrière l'école, en promenade [...] jusqu'à ce que toute l'école soit empoisonnée » (cité par Michal Jareš, op. cit., notre traduction).

56. Matthieu Letourneux, "Le genre comme pratique historique et médiatique (1860-1940) », Les Genres du roman au XIXe siècle, dir. Émilie Pézard et Valérie Stiénon, Paris, Classiques Garnier, coll. Rencontres, à paraître.

57. Matthieu Letourneux, «La disparition du genre des mystères au début du XX $\mathrm{X}^{\mathrm{e}}$ siècle », op. cit.

58. Pour la Hongrie, voir par exemple F. P., «Hands off !», op. cit. et Schöpflin Aladár, « Nick Carter ; Beszélgetés egy szép asszonnyal », Nyugat, 1908, 24, dans lequel le narrateur s'entretient avec une lectrice de Nick Carter, dément les soupçons d'un caractère criminogène de ce type de récits (preuve que la série avait au moins entraîné un début de panique morale dans ce pays), mais pour mieux condamner le caractère industriel de ce type de productions. Pour la Tchécoslovaquie, voir Michal Jareš, op. cit. qui montre bien l'effet de sidération produit par la modernité du support.

\section{RÉSUMÉS}

$\mathrm{Si}$, dans les imaginaires de la culture populaire, ce qui importe, ce sont les dynamiques architextuelles et leur formulation dans les séries culturelles et médiatiques, alors la question de la circulation internationale des imaginaires et de leur mondialisation doit se penser à un niveau plus global, en jouant en permanence entre le texte et son contexte matériel, parce que c'est ce contexte, autant que le texte, qui produit de la signification. C'est ce que montre l'exemple de l'arrivée des dime novels en Europe au début du XXe siècle, lesquels ont imposé partout en 
quelques années des genres de récits et des formats spécifiques. En effet, si ces séries américaines ont bouleversé les imaginaires européens, c'est moins grâce aux thèmes du détective et du cowboy que par la rupture structurelle entraînée par l'arrivée du format même du dime novel, entraînant de nouveaux modes de lecture et de nouvelles relations au texte. Même dans des espaces plus à la marge des mouvements de mondialisation, où ils ont été limités dans leur diffusion, souvent expérimentés de manière plus tardive, et absorbés en particulier par des logiques plus anciennes, ces phénomènes ont été malgré tout perçus comme l'expression d'une mutation culturelle majeure, associée à une marchandisation et à une industrialisation de la littérature, contribuant, dans ces espaces aussi, à la mondialisation des imaginaires.

If, in popular culture, what matters are the architextual dynamics and their formulation in the cultural and media series, then the question of the international circulation of imaginations and their globalization must be thought of on a broader level, by articulating the level of the text and that of its material context, because it is this context, as much as the text, which produces meaning. This is illustrated by the example of the arrival of novel dime in Europe at the beginning of the 20th century, which imposed in a few years everywhere kinds of stories and specific formats. Indeed, if these American series have upset European imaginations, it is less because of the themes of the detective and the cowboy than by the structural rupture brought about by the arrival of the format of the dime novel, leading to new modes of reading and new relationships to text. Even in European spaces more on the margins of globalization movements, where the distribution of these publications has remained limited, often experienced later, and absorbed by older logics, these phenomena were nevertheless perceived as the expression a major cultural mutation, associated with the commodification and industrialization of literature, contributing, in these spaces, also to the globalization of imaginations.

\section{INDEX}

Mots-clés : Europe, littérature populaire, culture médiatique, dime novel, fascicules, Eichler, Allemagne, France, Tchécoslovaquie, Hongrie, Norvège, Suède, 20e siècle, Nick Carter, Buffalo Bill Keywords : Europe, popular literature, media culture, dime novel, booklet, Eichler, Germany, France, Czechoslovakia, Hungary, Norway, Sweden, 20th century, Nick Carter, Buffalo Bill

\section{AUTEUR}

\section{MATTHIEU LETOURNEUX}

Matthieu Letourneux est Professeur à l'Université Paris-Nanterre et membre du CSLF. Il est rédacteur en chef de la revue Belphégor (https://journals.openedition.org/belphegor/). Spécialiste des cultures sérielles et médiatiques des XIXe et XXe siècles et de la littérature pour la jeunesse, il a publié un essai sur la sérialité, Fictions à la chaîne, Seuil, « Poétique », 2017. Il a fait paraître auparavant Cinéma, premiers crimes (avec A. Carou, Paris Bibliothèques, 2015), Fantômas! Biographie d'un criminel imaginaire (avec L. Artiaga, Les Prairies ordinaires, 2013), La Librairie Tallandier, Histoire d'une grande maison d'édition populaire (1870-2000) (avec J.-Y. Mollier, Nouveau Monde, 2011) et Le Roman d'aventures, 1870-1930 (PULIM, 2010).

Il a dirigé ou codirigé une dizaine de publications collectives sur les littératures de jeunesse ou les cultures médiatiques (derniers en date : L'Anticipation dans les discours médiatique et sociaux avec V. Stiénon, et Mutations des légitimités dans les productions culturelles contemporaines, Belphégor, 17, 1, 2019, avec Anne Besson, Anne Isabelle François, Sarah Lécossais, Matthieu Letourneux et Anne-Gaëlle Weber, https://journals.openedition.org/belphegor/1450). 
Il a réédité Le Coureur des bois de Gabriel Ferry (Phébus), On vole des enfants à Paris de Louis Forest (Le Masque), et chez Bouquins les œuvres de Gustave Aimard, Emilio Salgari, Eugène Sue et la série Fantômas (avec L. Artiaga). 